\title{
Las monedas sociales en la Economía Social y Solidaria: más allá de la reciprocidad, entendiendo las prácticas concretas a la luz del caso de "La Bellota"1
}

\author{
Gema Alcañiz
}

Recibido: 2 de febrero de 2019/ Aceptado: 8 de abril de 2019

Resumen. Este artículo se basa en un trabajo etnográfico sobre las llamadas "monedas sociales", a saber, aquellos dineros que solo se utilizan en ciertas prácticas y circuitos sociales restringidos que se han multiplicado en los últimos años, y, en concreto, en el caso de la experiencia de "la Bellota", la comunidad de intercambio creada en la ciudad de Guadalajara. El material etnográfico que se presenta lanza un desafío al tratamiento realizado sobre estas prácticas desde el marco de la Economía Social y Solidaria, en concreto, en lo referente al tipo de relaciones sociales que sustentan los intercambios en estos circuitos. Para ello, se propone un re-examen de un concepto clave para la Antropología, la noción de 'reciprocidad', muy subrayado en los modelos económicos alternativos que promueven estas experiencias, cuya aplicabilidad es cuestionable para el análisis de las mismas.

Palabras clave: Economía social y solidaria; dineros sociales; etnografía; reciprocidad; mercado.

\section{[en] Social money in the Social and Solidarity Economy: beyond the reciprocity, understanding the concrete practices in light of the case of "La Bellota"}

\begin{abstract}
This article is based on an ethnographic work on the so-called "social currencies", namely, those monies only used in certain practices and restricted social circuits that have multiplied in recent years, and in particular, the experience of "La Bellota", the exchange community created in the city of Guadalajara. The submitted ethnographic material issues a challenge to the treatment carried out on these practices within the framework of the Social and Solidarity Economy, specifically, with regard to the type of social relations that underpins the exchanges in these circuits. To do this, a re-examination of a key concept to Anthropology is proposed, the notion of 'reciprocity', very underlined in the alternative economic models that promote these experiences, whose applicability is questionable for the analysis of them.
\end{abstract}

Keywords: Social and solidarity economy; social money; ethnography; reciprocity; market.

Sumario. 1. Introducción: las monedas sociales a la luz de la Economía Social y Solidaria. 2. Marco conceptual: más allá de la reciprocidad. 2.1. Concepto y proyecto político. 2.2. Más allá de la reciprocidad: la articulación de lógicas económicas. 3. Hallazgos etnográficos. 3.1. Panorama general. 3.2. "La Comunidad de Intercambio de la Bellota": ejemplo de sistema de intercambio basado en crédito mutuo. 4. Discusión: Entre la reciprocidad y el mercado. 4.1. Después de la dicotomía clásica. Coexistencia de lógicas económicas. 5. Conclusiones. 6. Bibliografía.

\footnotetext{
1 El trabajo forma parte de una investigación etnográfica en el marco de una tesis doctoral que se está llevando a cabo en la UNED, bajo el "Programa de Doctorado de Diversidad, Subjetividad y Socialización. Estudios en Antropología social, historia de la psicología y de la educación".

$2 \quad$ Universidad Nacional de Educación a Distancia (UNED). galcaniz1@alumno.uned.es
} 
Cómo citar: Alcañiz, G. (2019). Las monedas sociales en la Economía Social y Solidaria: más allá de la reciprocidad, entendiendo las prácticas concretas a la luz del caso de "La Bellota", en Revista de Antropología Social 28(2), 275-295.

\section{Introducción: las monedas sociales a la luz de la Economía Social y Solidaria}

La denominada "Economía Social y Solidaria" (en adelante ESS) ha gozado de un indiscutible protagonismo en los últimos años, como demuestra su reconocimiento -tanto a nivel nacional como internacional- dentro del amplio abanico de propuestas económicas presentadas como "alternativas" o "transformadoras". De las numerosas iniciativas que pueden enmarcarse bajo el paraguas conceptual que esta propone, podemos destacar las llamadas monedas sociales, debido, en parte, a su espectacular florecimiento tras el estallido de la crisis financiera. En su escenario posterior, ligado también con otros movimientos sociales surgidos en torno al 15M de 2011, se empieza a apostar no solo por la construcción de prácticas económicas de carácter formal, sino también por crear todo un circuito de aprovisionamiento fuera del mercado, donde se incluyan fórmulas informales y no monetarias (bancos de tiempo, monedas sociales, redes de trueque, huertos urbanos, etc.).

En estos casos se pretende la creación de redes, principalmente entre desconocidos, como fórmula de cohesión dentro de una comunidad y como estrategia de abastecimiento de recursos donde no prime la lógica de compra-venta del mercado. El potencial que se atribuye a las prácticas económicas de tipo informal ${ }^{3}$ y no monetario, reside precisamente en ofrecer una escapatoria a la dependencia del dinero oficial como única vía de acceso a los recursos de vida necesarios.

Dado que a las monedas sociales se les otorgan los mismos principios que guían a la ESS, el objetivo de estas, según sus defensores, es ubicar a las personas y a su entorno natural ${ }^{4}$ en el centro de los procesos económicos (o la reproducción de la vida, propósito principal de la ESS). En este orden de cosas, ha sido notable el interés suscitado en el ámbito académico por las monedas sociales como parte de la propuesta de la ESS, si bien la literatura encontrada proviene principalmente de los sectores más involucrados en la promoción de estas prácticas, existiendo una frontera un tanto difusa entre teóricos y lo que podríamos considerar "activistas".

La noción de "moneda social" fue utilizada por primera vez por Primavera, reconocida activista dentro del movimiento en defensa de estas monedas. Con ella, se hacía alusión a las experiencias de los Clubes de Trueque que emergieron en Argentina, con el fin de destacar "sus efectos de inclusión social" (Primavera, 1999). Asimismo, se interpreta el apellido "social", en relación a las supuestas funciones sociales que deja de lado el dinero convencional por sus características favorables a la acumu-

3 Aun así, el mayor énfasis de este modelo "alternativo" se encuentra asociado al potencial de la creación de una red sólida de empresas cooperativas en el ámbito formal, en todas las fases del proceso económico, producción, distribución y consumo. En las prácticas de carácter informal se puede observar claramente un marcado carácter ideológico, al pretender una emancipación del mercado en su anhelo de un "nuevo" sistema económico.

4 Los objetivos ecológicos que persiguen las monedas sociales son frecuentemente resaltados en los estudios sobre el tema. En general, frente al modelo de crecimiento dominante y el sistema monetario convencional, la mayoría de los autores se muestra optimista acerca de las facultades sostenibles de estas monedas, que al no estar basadas ni en el dinero deuda ni en el interés compuesto, libera a los sistemas económicos del imperativo del crecimiento perpetuo (Corrons, 2015; Yasusuki, 2017). 
lación y su escasez (Gisbert, 2010). También, en referencia a su implementación y gestión, mayormente dependiente de organizaciones locales y de base comunitaria (Blanc, 2011). Pero igualmente son conocidas como monedas alternativas, comunitarias, complementarias, locales y/o paralelas, unas veces de manera indistinta, otras para remarcar las diferentes pretensiones de cada modelo existente.

Llegados a este punto conviene destacar que de manera rigurosa se denomina moneda a un tipo de dinero - una pieza material, generalmente acuñada por alguna autoridad o su equivalente en papel- que es utilizado como medio de cambio. Sin embargo, el dinero puede adoptar otras formas no necesariamente materiales -como sucede en muchas ocasiones con el dinero fiduciario- porque se trata de todo medio de intercambio común y generalmente aceptado por una sociedad para el pago de bienes, servicios y obligaciones de cualquier tipo. Basándonos en esta definición, y en sentido estricto, en el caso que estamos analizando, sería más preciso hablar de "dinero social" que de "monedas sociales", dado que en múltiples ocasiones este tipo de sistemas de intercambio no utilizan ningún objeto material que haga las veces de "moneda" sino que se apoyan en diversas formas de contabilidad -como cartillas o diversos sistemas informáticos-, basados en la confianza entre los diferentes miembros de la comunidad. No obstante, aquí hablaremos de "monedas sociales" por ser el término que más se ha popularizado. Esta etiqueta esconde fenómenos de muy diversa índole como veremos más adelante.

En cualquier caso, con esta explicación se pretende remarcar que, más allá del uso que los participantes hacen de la noción "moneda social" como una categoría "emic", el concepto hace referencia a una forma de dinero5. Lo que caracteriza al mismo es su uso exclusivo como medio de intercambio en ciertos circuitos sociales restringidos fuera del mercado. A diferencia del dinero como medio de cambio generalizado en la esfera de mercado, este representa un poder adquisitivo restringido y queda circunscrito a una red limitada y específica de bienes y servicios en la que su uso tiene cabida.

Del mismo modo, un elemento clave de la definición recae en los objetivos que se proponen. A este respecto se considera que las monedas sociales "promueven la reciprocidad y la ayuda mutua, contribuyendo a la articulación de comunidades solidarias" (Santana, 2009:6). Se considera, pues, que la participación en estos grupos puede facilitar la construcción de nuevos vínculos sociales capaces de transformar la lógica mercantil de los intercambios (Orzi, 2009). En este sentido, uno de los propósitos principales es cambiar la relación que establecen las personas, "al modo de 're-incrustar' las transacciones en una relación humana más amplia, promoviendo así el desarrollo de relaciones interpersonales mediante la convivencia y la amistad" (Blanc, citado en Orzi, 2009) ${ }^{6}$. Estos autores presagian, por otro lado, que un uso no capitalista del dinero fortalecerá un tipo de relaciones socioeconómicas diferentes en virtud de la reproducción de otro tipo de sociedad y economía (Orzi, 2009).

5 Para entender su significado conviene hacer uso de la importante distinción que realizase Polanyi entre "dinero para todo uso y dinero para usos especiales". Esta separación sirve para denotar la diferencia entre el objetodinero que se generaliza para todo uso al servir para las cuatro funciones atribuidas al dinero (medio de cambio, patrón de valor, depósito de riqueza y medio de pago), a diferencia de los objetos-dinero que sirven solo a uno o varios empleos, restringidos por norma a ciertos usos especiales o específicos.

6 A este respecto, Blanc (2011), autor de una de las tipologías más ampliamente reproducida, sitúa a las monedas sociales dentro de la categoría de proyectos de naturaleza comunitaria que buscan fortalecer la comunidad bajo el principio director de la reciprocidad. 
En esta misma línea, otro concepto que deviene central en la teoría de las monedas sociales es el de capital social -una interpretación singular de la reciprocidad. Algunos planteamientos de este concepto utilizan el término para referirse a las relaciones sociales que caracterizan los ámbitos de actuación de este tipo de experiencias (comunitarios, de red, etc.). Los teóricos inciden en que la participación en estas experiencias aumenta el capital social de las personas, basándose principalmente en dos indicadores: por un lado, la proliferación de interconexiones y redes, y, por otro, el comportamiento de la comunidad, que, inspirado por las normas de reciprocidad, deviene en cooperación ${ }^{7}$ (Seyfang y Longhurst, 2013; Oliver, 2013). En cualquier caso, en estos estudios no se transmite una noción clara de cómo son en realidad las relaciones que se dan.

Estas iniciativas proponen una "alternativa" a la circulación del mercado, y al tipo de relaciones que se asocian a este último, y tratan de fomentar relaciones sociales de reciprocidad bajo el nombre de solidaridad. Pero ¿estas realidades económicas y sociales complejas pueden ser analizadas sin problemas mediante la aplicación del concepto antropológico de reciprocidad?, ¿en qué sentido pueden ser consideradas fuera de la lógica de mercado? Desde el marco de la ESS, los trabajos existentes sobre estas prácticas refuerzan una visión dicotómica entre dos lógicas económicas contrapuestas. Pensamos que es necesaria una problematización de esta división dicotómica, puesto que esta literatura reproduce un significado de las relaciones sociales disociado de su realización concreta en las prácticas existentes.

Para atender a las cuestiones anteriores, en este artículo se propone un re-examen del concepto de reciprocidad a la luz del estudio realizado en la red de intercambio de la Bellota, pero antes es fundamental hacer una breve reflexión sobre las relaciones que parecen tan centrales en la propuesta de la ESS y las monedas sociales, a fin de aclarar algunas cuestiones sobre las posibles limitaciones de la aplicación de este concepto teórico, además de señalar el proyecto político - pasado y actual-detrás de esta noción.

\section{Marco conceptual: Más allá de la reciprocidad}

\subsection{Concepto y proyecto político}

El concepto de reciprocidad es una noción central del análisis antropológico que describe formas de interacción social que no están guiadas por la lógica del mercado. No obstante, su interpretación, y uso, no han estado exentos de problemas dentro de la propia disciplina, dada la imprecisión inherente a este concepto para describir un tipo de relaciones complejas (Narotzky, 2016).

Pero, siguiendo a esta autora, para comprender la problemática inmanente a la aplicación de este concepto conviene una relectura de los autores principales del mismo, remontándonos al contexto histórico de su producción. Mauss muestra cómo la reciprocidad, esa mezcla de interés material y obligación moral que sustenta un

\footnotetext{
Estos autores siguen a Putman (1993) en su vaga definición de capital social como "aquellos aspectos de la organización social, como la confianza, las normas y redes, que pueden mejorar la eficiencia de la sociedad al permitir la acción coordinada" (Putman, 1993:167). Lo curioso de esta definición es la "libre" disposición a cooperar que el capital social parece incentivar.
} 
sistema complejo de transferencias que producen solidaridad y cooperación, es el núcleo de la cohesión social. Pese a que Mauss nos remite en su análisis a la particularidad $^{8}$ de estos sistemas de intercambio en las sociedades primitivas, no obstante la teoría que presenta (de la reciprocidad como cemento de la sociedad) se establece como una teoría universal de la cohesión social, en la cual la reciprocidad ha resultado un concepto de uso general que puede implementarse en otros contextos (Narotzky, 2016). Así, desde su origen se construye como un concepto abstracto que, no obstante, está pensado para describir relaciones sociales por definición específicas -históricas, culturales, sociales, etc. De ahí que nos parezca pertinente la revisión de la teoría de la reciprocidad al señalar que precisamente son los ámbitos concretos los que pueden dotar de sentido a la misma.

En sus conocidas conclusiones del Ensayo sobre el don, Mauss establece un vínculo directo entre el análisis del don en las sociedades primitivas y arcaicas y el proyecto político-moral de revitalizar este tipo de relaciones, como ahora recupera la ESS. Como señala Mauss:

Por lo tanto, podemos y debemos volver a la sociedad arcaica y a los elementos que la integran. Encontraremos en estas razones de la vida y la acción que aún prevalecen en ciertas sociedades y numerosas clases sociales: la alegría de las donaciones públicas; el pleito en gastos generosos en las artes, en la hospitalidad y en el festival privado y público (2009: 236).

El concepto de reciprocidad que desarrolla Polanyi contrasta con la visión de Mauss, quien formula la reciprocidad en un sentido estructural como una de las formas de integración. La característica principal de estas es que la organización de los intercambios se fundamenta en una simetría entre las partes, es decir, que la reciprocidad se ejerce entre iguales. En la formulación del don expuesta por Mauss, no obstante, si bien este puede hacer referencia a movimientos de bienes recíprocos, el grueso de su teoría se fundamenta en datos provenientes de sociedades jerárquicas, por lo que el don ${ }^{9}$ también se integra en relaciones de tipo redistributivo entre jefes y otras autoridades. La propuesta de Polanyi permite una mayor flexibilidad para ampliar el análisis a diferentes contextos, y permite situar el intercambio recíproco en articulación con el resto de principios económicos presentes en la sociedad (la redistribución y el intercambio de mercado). Igualmente, para este autor, más allá de la consideración de la reciprocidad como una forma de integración, es también considerada un modelo de lo que son o deberían ser las relaciones sociales apropiadas en cada cultura (Moreno, 2011).

Aparece de un modo bastante claro la vinculación de la ESS con estos autores en tanto que reivindican en la actualidad una re-moralización de la economía -un reclamo de los objetivos humanos que han de prevalecer en los procesos económicos

8 La definición de Mauss de la reciprocidad en base a tres obligaciones - dar, recibir, devolver- es restrictiva en el sentido de que su utilidad se encuentra limitada a ciertos ámbitos de la vida social, como la amistad y el de la hospitalidad, siempre y cuando se dé en ellos el valor moral de la generosidad (Sanz, 2002).

9 Recordemos aquí que el tema principal del Ensayo es la circulación de los dones, las obligaciones entre los grupos que dan, reciben y devuelven y el total de relaciones que extensivamente traban las partes implicadas, entre las que hay cabida no solo para la concordancia, sino también para el conflicto y la desigualdad (Moreno, 2011). 
o una re-incrustación de la economía en la sociedad (Narotzky, 2010). A la luz de estas filiaciones se evidencia, por tanto, la intención de estos proyectos actualmente.

Como vemos, el nacimiento de este concepto está asociado a proyectos políticos que abogarían por un equilibrio, al estilo de una "Tercera Vía" o "economía mixta", y que rehuirían tanto de un contexto de capitalismo incontrolado como de un socialismo de Estado, similar al solidarismo francés del S.XIX (Narotzky, 2010). La ESS, en su proyecto actual, retoma la noción de solidaridad en tanto que "cemento" necesario para cohesionar la sociedad, y lo readapta a la ideología de la lucha por la transformación social actual - presente también en el movimiento anterior (Alquézar, 2015). Sin embargo, si bien en el contexto precedente se remitía a la cooperación en términos de la clase -la solidaridad obrera- como valor contrapuesto a la presión del mercado, el principio de solidaridad ahora, en lugar de remitir a un orden de obligaciones morales dentro de una misma comunidad, se asocia a lo que Laville denomina una reciprocidad voluntaria ${ }^{10}$, a saber, un principio de asociación libre y voluntario entre personas (Laville, 2004).

Si bien activistas y teóricos de la ESS, subrayan la importancia de fomentar relaciones sociales de reciprocidad bajo el nombre de solidaridad, se pretende aquí advertir de los peligros de una utilización mecánica de este término para referirse a las relaciones sociales que caracterizan estos ámbitos, entre los que se encuentran las monedas sociales, como ejemplo de estas iniciativas solidarias, dado que el concepto clásico pierde valor analítico cuando se aplica indiscriminadamente. De acuerdo con Narotzky (2016), consideramos que aquí puede residir una de las principales dificultades del realismo etnográfico en este tipo de prácticas. Esto nos lleva a comprender simultáneamente dos niveles de realidad distintos: por un lado, las relaciones que se pueden encontrar en estas experiencias y, por otro, el papel que juega la reciprocidad -y el ideal de su aplicación- en los proyectos políticos en curso.

\subsection{Más allá de la reciprocidad: la articulación de lógicas económicas}

Las interpretaciones vigentes en la literatura, a su vez, favorecen la construcción de una visión dicotómica y esencializadora de las relaciones económicas prevalecientes en sociedades divididas como primitivas/modernas, que dificulta la comprensión de relaciones complejas que aparecen en las situaciones empíricas. Como hemos visto, tanto Mauss como Polanyi reforzaron esta dicotomía clásica de relaciones sociales "extraeconómicas", a las que era recomendado retornar, frente a relaciones definidas como "económicas", al señalar la reciprocidad como un concepto referido a lo opuesto a las relaciones del intercambio de mercado. Pero, frente a esta supuesta escisión, como señala Narotzky:

El concepto de reciprocidad puede ayudarnos a analizar unas relaciones ubicuas en la realidad económica y permite explorar la ambivalencia originaria ente "interés" y "moral" pero no como algo "exterior"11 y "anterior" al "mercado" sino

10 De algún modo esta reciprocidad voluntaria podría asemejarse a la "cooperación voluntaria" destacada en el concepto de capital social propuesto por Putman anteriormente.

11 Esta visión está muy presente en autores como Harvey (2004), quien subraya esta idea en su concepto de "acumulación por desposesión", considerando la dialéctica del dentro-fuera que rige la acumulación del capital, en la que el "afuera" -que no es naturalmente externo ni anterior- ha de ser continuamente creado por el sistema 
como precisamente algo constitutivo de este sistema (...), permite observar relaciones sociales situadas simultáneamente en circuitos de aprovisionamiento dentro y fuera del mercado (Narotzky, 2010:154).

Sin embargo, los antropólogos clásicos no desmintieron que, en las sociedades estudiadas, se diesen "intercambios de mercado"12 (gimwali o trueque de productos básicos) aunque considerados de escaso valor en comparación con otro tipo de intercambios relacionados con el prestigio social como el kula (Malinowski, 1986 [1922]). Del mismo modo, tanto el dominio de la entrega de dones en las sociedades precapitalistas como la ubicuidad de la norma del mercado en las sociedades contemporáneas, deben ser cuidadosamente considerados y cuestionados (Godelier, 2004). Numerosas etnografías han demostrado que el comportamiento de dar regalos, junto con otros tipos de transacciones no monetarias, también pueden detectarse en contextos capitalistas (Bloch y Parry, 1989).

Para estos autores, la amplia gama de transacciones que se producen en una sociedad deben comprenderse en el marco más amplio de la reproducción social (Bloch y Parry, 1989). Con todo, plantean la coexistencia de diferentes lógicas económicas basándose en parte en la idea de economía multicéntrica con esferas de intercambio ${ }^{13}$ separadas pero interrelacionadas de Bohannan (1981). Desde este punto de vista, distinguen entre dos esferas $-\mathrm{u}$ órdenes transaccionales-: por una parte, las transacciones relacionadas con la reproducción del orden social a largo plazo -ámbito de la comunidad-; por otro, una esfera de transacciones a corto plazo relacionadas con el ámbito de la competencia individual -ámbito del comercio (Bloch y Parry, 1989).

En este sentido, y en relación a los estudios sobre el dinero contemporáneo, Zelizer (2015) ofrece también una crítica a la teoría de las esferas separadas que prima en los análisis sociales (con base en principios rivales e incompatibles: sentimientos y racionalidad, solidaridad y egoísmo, etc.), y propone un marco de coexistencia e interacción entre las transacciones de mercado y las relaciones personales. Aplica la noción antropológica de esferas de intercambio al estudio de las monedas sociales que identifica con la noción de "circuitos de comercio restringidos"14. Estos circuitos, según la autora, funcionan, en el seno de las sociedades capitalistas, como puente para facilitar la convivencia de lazos sociales íntimos e impersonales. Zelizer identifica estos circuitos con base en los siguientes elementos: 1) tienen una frontera

capitalista. Este marco implica entender la articulación de diferentes lógicas económicas en el contexto de hegemonía capitalista vigente.

12 Aquí es necesario traer la distinción teórica y empírica establecida por Polanyi entre, por un lado, "mercado" como lugar de mercado o emplazamiento específico donde se encuentran un grupo de compradores y vendedores, presente en algunas sociedades "primitivas", al que nos referimos aquí, y por otro, "sistema de mercado" como mecanismo de oferta y demanda que rige todo el proceso productivo, cuya aplicación a las sociedades no integradas en el capitalismo, no es posible.

13 Esta noción está íntimamente relacionada en la Antropología a la distinción de Polanyi mencionada anteriormente entre "dinero para todo uso y dinero para usos especiales", estos últimos asociados a diferentes esferas de intercambio en las economías multicéntricas.

14 Aunque no ahonda en la naturaleza de su creación, estamos de acuerdo con la autora en señalar que no se pueden ignorar los contextos socioeconómicos y políticos en el surgimiento de estos proyectos, como pueden ser la falta de respuesta institucional ante determinadas problemáticas económicas y sociales, o la ausencia de provisión de determinados bienes y servicios por parte de las autoridades competentes, en un contexto de recortes en gastos destinados a las políticas sociales. En este sentido, las personas optan por esta suerte de estructuras basadas principalmente en la auto-organización. 
bien definida con cierto control sobre las transacciones que cruzan sus límites, 2) entre sus lazos interpersonales tiene lugar un conjunto distintivo de transferencias de bienes, servicios o reivindicaciones de derechos sobre ellos, 3) dichas transferencias emplean medios distintivos, y 4) los lazos entre participantes tienen significados compartidos (Zelizer, 2015: 384).

El propósito ahora, a la luz de un trabajo etnográfico aún en curso sobre una experiencia de moneda social, es comprobar la idoneidad del concepto de reciprocidad para el análisis de estas redes, siempre y cuando se tome la reciprocidad en su articulación con el mercado dentro del contexto estudiado.

\section{Hallazgos etnográficos}

\subsection{Panorama general}

Las monedas sociales han tenido un extraordinario crecimiento en los últimos años, proliferando en numerosas localidades y países, si bien con diversas particularidades y diferencias. Paralelo a la profundización de la crisis económica, el aumento del desempleo y la desindustrialización, hemos asistido a un importante auge de este tipo de iniciativas, como lo prueba el hecho de que en la plataforma de intercambio mundial "Community Exchange System" (https://www.community-exchange.org) casi el 30\% de las iniciativas registradas sean españolas ${ }^{15}$. Si bien la aparición en el caso español de estas experiencias socio-económicas está vinculada al estallido de la crisis en el año 2007, no se puede obviar el contexto previo de crisis generalizada y de desmantelamiento del Estado de bienestar, que nos pone en la pista sobre el modo en que han surgido iniciativas parecidas en países como Argentina o Reino Unido -Clubes de trueque o LETS, respectivamente (Sanz, 2002).

Bajo su aparente novedad, las monedas sociales actuales gozan de un largo recorrido dentro de la historia, ya que la presencia de dineros alternativos ha sido muy frecuente en numerosos contextos de crisis históricas, como en las guerras -vales y cupones- o durante la gran depresión norteamericana de la década de 1930, donde se pusieron en práctica muchos sistemas de intercambio en zonas muy castigadas por la crisis económica. En esta dirección destacan algunos experimentos de dineros sociales basados en las teorías de Silvio Gesell, como el de Wörgl (Austria), donde se pusieron en circulación certificados de trabajo o pagarés sellados. Este dinero tenía la particularidad de aplicar una tasa de oxidación, que depreciaba el valor del dinero un tanto por ciento cada mes para incentivar su uso y evitar la acumulación del mismo. Pese a todo, las autoridades gubernamentales, el banco central y el tribunal supremo austríacos acabaron prohibiendo este dinero por considerarlo ilegal.

La mayoría de los proyectos actuales adaptan o mezclan distintos fenómenos presentes en otros momentos históricos, como pueden ser cupones utilizados como medio de pago basados en el valor del dinero oficial, los registros de los intercambios al modo de los bancos de tiempo o los que tienen como base el intercambio de mercancías. Desde un punto de vista antropológico, más que de innovaciones

15 Un mapa sobre la situación de las monedas sociales existentes en España, y en todo el globo, puede encontrarse aquí: https://mapo.komun.org/. Por otro lado, el siguiente blog http://www.vivirsinempleo.org/ es seguramente una de las principales referencias con información sobre todas estas iniciativas. 
alternativas se trata de aplicaciones actuales de viejas prácticas muy conocidas en distintos contextos y países. En todo caso, la novedad de los dineros sociales actuales consiste en su vigor dentro de los movimientos sociales que buscan alternativas a las economías nacionales y globales, y reivindican el derecho a crear su propio dinero al margen de cualquier autoridad o entidad financiera que mantenga fuera de su control el manejo del mismo. Diferentes autores han puesto, además, el acento en las potencialidades que internet ofrece precisamente para la creación de instrumentos financieros que sirvan a los intereses de las personas, en aras de lograr unas mayores cotas de democracia económica y para la innovación de nuevas formas de dinero, que permitan prescindir de intermediarios tradicionales como los bancos (Hart, 2001; Lanchester, 2016).

La mayor parte de los estudiosos sitúan el surgimiento de estos "nuevos" sistemas de intercambio en los años 80 con la creación del primer LETS (Local Exchange and Trade System) en Canadá (Vancouver), en respuesta a un contexto de crisis generalizado dentro de la economía local de este municipio (Gisbert, 2010). Con el tiempo, la idea ha sido extendida también en Australia, Inglaterra, Francia (denominada SEL, o Système d'Echanges Locaux) y Alemania (Tauschring). Se trata de sistemas de intercambio de bienes y servicios a escala limitada -local- que utilizan una moneda o divisa propia, sin existencia física. Entre sus características principales destacan las siguientes: 1) la suma de todas las cuentas es siempre cero, es decir, algún usuario tiene que quedarse con saldo negativo para que otros estén en saldo positivo. El registro de las transacciones se realiza manual o electrónicamente; 2) no se cobra ninguna tasa de interés a los saldos negativos: el deudor puede cancelar su saldo cuando quiera con lo que tiene (productos) o con lo que sabe/puede hacer (clases de inglés, fontanería) sin tener que pagar una tasa de interés (Yasuyuki, 2017). Numerosas experiencias de moneda social actuales recurren a los esquemas de tipo LETS para la gestión de los intercambios en la red.

A pesar de la existencia de numerosas variaciones en torno a los proyectos de moneda social, los análisis de estas permiten establecer algunas distinciones, principalmente en torno a los objetivos centrales que persiguen y los mecanismos en uso en cada una de estas iniciativas ${ }^{16}$ (Lietaer, 2005, Gisbert, 2010). En relación a los objetivos, aunque las monedas sociales ofrecen un amplio abanico de opciones económicas y sociales, tratando de poner un poco de orden al respecto, se distinguen, por un lado, las que persiguen objetivos con un carácter más claramente económico, como la activación del tejido comercial local, y, por otro, las enfocadas a objetivos sociales, como crear lazos entre los miembros de una comunidad (Lietaer, Kennedy y Rogers, 2015). Si bien entre el primer grupo se da frecuentemente la colaboración de pequeñas empresas, tiendas o negocios, incluso instituciones, el segundo tipo suele conformarse especialmente por particulares que no responden en principio a ninguna entidad empresarial o administrativa.

En relación a los mecanismos en uso existentes, la mayoría de autores identifica una serie de fenómenos bastante diferentes entre sí, pero, siguiendo a Zelizer (2015), serían; 1) monedas con tipo de cambio fijo, 2) intercambios de tiempo, 3) trueque, y 4) sistemas basados en bienes de consumo, o sistemas de crédito mutuo -como son

16 Un aspecto fundamental en juego a la hora de distinguir entre las diferentes propuestas de monedas social es la naturaleza de su emisión, bien partan de alguna autoridad (consistorios municipales en colaboración con Organizaciones No Gubernamentales), empresa y/o la propia ciudadanía. 
conocidos mayormente- ${ }^{17}$. A este último grupo se adscribe la experiencia que se presenta a continuación y la mayoría de las experiencias que han surgido en el territorio español durante los inicios de la crisis económica, aunque en el momento actual se observa un despunte de iniciativas con respaldo institucional (como la creada en Barcelona en octubre de 2018 con apoyo del consistorio municipal).

La etiqueta "moneda social" (al igual que su correspondencia general, la ESS) contiene mezcladas posiciones teóricas divergentes, que no coinciden en sus objetivos y que están "incrustadas" en diferentes proyectos políticos definidos como "alternativos".

\section{2. "La Comunidad de Intercambio de la Bellota": ejemplo de sistema de intercambio basado en crédito mutuo}

El material etnográfico aquí presentado se centra principalmente en la experiencia de la Comunidad de Intercambio de Guadalajara, la Bellota. El proyecto surge en el año 2013 por parte de un grupo de personas interesadas en poner en práctica una iniciativa local de moneda social al calor de algunos contactos con integrantes de otras experiencias cercanas similares, como La Mora de la Sierra Norte de Madrid, surgida un año antes.

Su aparición está íntimamente ligada al espacio "El Rincón Lento", iniciativa creada en la misma ciudad en torno al año 2009 y que, en la actualidad, reúne alrededor de 300 familias. El Rincón Lento se autodefine como "un local multiespacio dedicado al consumo responsable, a la difusión cultural y la creación de tejido social". El propio nombre de la iniciativa da idea de la idiosincrasia de este espacio, que en torno a su reivindicación de "lo lento" construye, de una forma un tanto ambigua, su propia identidad y el carácter de las actividades que lleva a cabo. El Rincón Lento se presenta de la siguiente manera en un vídeo de su página web ${ }^{18}$.

Los movimientos que abogan por la lentitud buscan una vida más sosegada, y esto al final se va a reflejar en una actitud que cuida del medioambiente, que se preocupa por la sostenibilidad, que se preocupa por lo social, por las personas, por trabajar en unos términos económicos más justos, mucho más ético (...) principalmente porque queríamos que fuese una alternativa a la rapidez que nos impone el sistema y el modo de vida actual, y también porque, en muchos aspectos, nos sentimos identificados con el movimiento slow, que es un movimiento más general que tiene mucha de esta filosofía. Nos define nuestra manera de hacer las cosas, pensamos que los medios son tan importantes como los fines, entonces nuestros procesos, nuestras actividades tienen unos medios bastante justos y sostenibles con el entorno, con el medioambiente.

Este espacio se ha constituido en un referente en Guadalajara, aglutinador de diversas iniciativas presentadas como "alternativas" (red de intercambio de objetos re-

\footnotetext{
17 La clasificación seguida por Zelizer (2015) se basa en la de Blanc (2011), la más reconocida dentro de los estudios sobre monedas sociales. Pero existen diferentes maneras de clasificar estas mismas realidades, según otros autores. Para un análisis más pormenorizado véanse por ejemplo Gisbert (2010) y Seyfang y Longhurst (2013).

$18 \mathrm{http}: / / w w w . e l r i n c o n l e n t o . o r g /$
} 
utilizados -Rincón ReUsa ${ }^{19}$-, grupo de consumo de agricultura ecológica, etc.), a la vez que es un punto de encuentro abierto a múltiples iniciativas sociales y culturales de muy diversa índole (talleres de reciclaje, charlas, cenador vegano, etc.). Además, se ha convertido en un lugar abierto a la experimentación, del que han nacido otros proyectos de iniciativa social que se han implementado también en la ciudad, tales como un espacio de co-working (Karaba), entre otros.

Dentro de este contexto general y a raíz de una mesa redonda sobre otras experiencias en monedas sociales, se constituyó un grupo promotor interesado en crear una iniciativa que favoreciese los intercambios a nivel local. Durante casi un año este grupo realizó una serie de reuniones con el fin de documentarse e inspirarse en diferentes experiencias ya existentes, y finalmente se optó por crear una moneda social propia.

Tras la redacción de los Términos y Condiciones de Uso y Funcionamiento de la misma, en octubre de 2013 esta experiencia daba sus primeros pasos con el nombre de la Bellota. En estos últimos se recogían los objetivos que perseguía la Bellota:

Pretendemos ser un espacio que ayude a construir una economía al servicio del bien común. Que dinamice la economía local y favorezca su relocalización, evite la acumulación y sea una herramienta que potencie los talentos y capacidades de las personas, fomentando la solidaridad y el apoyo entre sus miembros.

El modo de funcionamiento de la Bellota se inspira en los sistemas denominados LETS, cuyas características han sido descritas anteriormente. Dado que en este modelo no existe una circulación de billetes ni monedas, para el registro de los intercambios se utiliza la plataforma informática CES (Community Exchange System), un sistema que permite a cada socio llevar su propia contabilidad y anotar el registro de todas las transacciones realizadas en su cuenta. Este programa permite además conocer los movimientos y saldos de los demás miembros de la red.

La comunidad cuenta con cerca de 200 socios, con edades comprendidas mayoritariamente entre los 30 y 55 años, en su mayoría pertenecientes a la ciudad de Guadalajara y algunos pueblos cercanos.

Algunas de las características más destacables de la Bellota, se recogen en sus Términos y Condiciones de Uso y Funcionamiento ${ }^{20}$ :

- Las transacciones realizadas en bellotas se regirán por los mismos principios y normas legales que las normalmente realizadas en euros.

- Si la Asamblea General así lo decidiera, la Administración podrá cobrar impuestos a las operaciones realizadas, tanto en bellotas como una porción en euros de un intercambio.

- Inicialmente la cuenta de cada socio comienza con cero unidades de moneda local, pero se pueden realizar intercambios hasta un saldo negativo de -150 (débito) ${ }^{21}$.

19 Con el lema, ¡pide lo que necesitas! ¡regala lo que no usas! describe este grupo su objetivo en Facebook. Se trata de un espacio en dicha red social que pone en contacto a donantes y receptores de objetos, con el propósito de darles una segunda vida y salir de la espiral de consumismo del sistema actual, según señalan sus creadores (puede verse también en http://www.elrinconlento.org/)

20 http://labellotadeguada.blogspot.com/

21 Actualmente -y por decisión del grupo- se ha anulado el límite positivo. 
- Si un comprador excede el límite de débito será avisado y orientado a diversificar su oferta, y si persiste podrá ser rechazado por la Administración.

- Será el deber de cada vendedor en una transacción el asegurarse de que los compradores no han superado los límites al aceptar una transacción, avisando a la Administración, que mediará y reclamará si el comprador no responde.

- Las ofertas deben ser descritas con transparencia y honestidad en los anuncios. Los anuncios considerados ofensivos para los demás, inexactos, ilegales o contrarios a la ética del sistema podrán ser retirados por la Administración sin consulta previa.

- Una oferta anunciada en el sistema equivale a una invitación para hacer negocios y no constituye una oferta irrevocable.

Al estar inspirada en los sistemas LETS, tanto el mecanismo de la Bellota como el del resto de los sistemas denominados de "crédito mutuo", es muy similar a grandes rasgos al de cualquier Banco del Tiempo (BDT en adelante), que podríamos considerar como sus predecesores, aunque se introducen matices en algunos aspectos. En los BDT el intercambio se reduce normalmente a servicios, siendo la unidad de valor para medir los intercambios, el tiempo, valorado por igual en todas las transacciones. En cambio, las monedas sociales introducen la posibilidad de adquirir también productos y la unidad de valor para medir los intercambios es una moneda local consensuada por el grupo (aunque en ocasiones pueda ser también el tiempo), como pueda ser la Bellota, la turuta, la mora, el eco, etc. Las monedas sociales buscan en teoría valores distintos a los de las monedas nacionales en circulación en los mercados monetarios nacionales e internacionales, por lo que incluso algunas de ellas plantean una alternativa más radical en relación a la medición del valor de los intercambios ${ }^{22}$.

El dinero en forma de crédito se emite por medio de las transacciones que se realizan dentro de la red, funcionando como un préstamo entre personas. Por lo general, estas monedas están en paridad 1:1 con las monedas nacionales, lo que, según los estudiosos, facilita su uso a la hora de poder valorar los bienes y servicios en relación a la esfera formal de la economía (Gisbert, 2010). Los bienes y servicios, de hecho, pueden valorarse de manera mixta en moneda social y moneda de curso legal. La paridad mencionada no implica convertibilidad alguna respecto al dinero de curso legal, ya que estos dineros normalmente están respaldados por los propios bienes y servicios que se ofrecen. Los intercambios no son bilaterales sino generalizados, es decir, las ofertas y demandas se entrecruzan dentro del grupo: cada vez que un miembro de la red requiere un servicio, la deuda no la contrae con quien se lo presta, sino con todos los miembros del grupo. En contadas ocasiones también puede ocurrir que

22 En la práctica, se refleja una tensión con respecto a la definición de las unidades de valor y las equivalencias de los intercambios. En algunas experiencias, al igual que es planteado en los BDT, el valor de algunos servicios como por ejemplo la impartición de talleres formativos, se valora según una tasa fija y es igual para todos los casos. Estas decisiones son acordadas en asamblea y dan lugar a reglas que no están exentas de debates en el seno de los grupos. Detrás de estas normas se encuentra la ideología de otorgar el mismo valor a todos los trabajos ofrecidos dentro de la red. Este tipo de iniciativas, de carácter más libertario, suelen excluir la participación de administraciones y empresas con asalariados (al considerar que esta fórmula laboral reproduce jerarquías). Lo que pretenden es crear una esfera lo más alejada posible del dinero convencional. Este sería el caso de experiencias como la Mora o la Canica. 
las transacciones se realicen sin medición de la moneda, es decir, que se produzca una compensación inmediata (o trueque). Como vemos, la cuantificación está muy presente en estas iniciativas, ya que los intercambios deben evaluarse de acuerdo con la noción de valor establecida, aunque pretenda esta ser independiente del valor de cambio que prevalecería en el mercado.

Las ofertas y demandas se publican en la plataforma informática CES, mencionada anteriormente. Entre las primeras destacan: ropa, complementos, artesanías, juguetes, cosmética natural, artículos de segunda mano, cursos de formación (de malabares, yoga, taichi, Pilates, etc.), trabajos de reparación y para el hogar, cuidado y talleres para niños, ofertas de ocio y turismo, terapias naturales, cuidados estéticos y de enfermería, acompañamiento y asesoramiento para personas mayores, servicios de diseño gráfico, coaching, defensa jurídica, mecánica de bicicletas, entre otros. Actualmente, además, se pueden comprar algunos productos de alimentación básica, de origen ecológico, en El Rincón Lento. Además, para facilitar los intercambios se ha creado un grupo cerrado en Facebook para aquellos miembros interesados, donde se publican casi a diario anuncios con ofertas y demandas. Esto, en ocasiones, genera malestar entre algunos participantes que no utilizan las redes sociales, al sentirse en desventaja. Por regla general, el perfil de estos últimos coincide con usuarios muy comprometidos dentro de la red, que animan constantemente a usar la plataforma oficial para que todo el mundo pueda tener las mismas oportunidades.

La gestión y dinamización de la red de intercambio genera tareas que, con frecuencia, dan lugar a la remuneración en bellotas. Por lo general, es el núcleo motor (un grupo no superior a diez personas) el que asume estos trabajos y se involucra en funciones de coordinación, mientras que el resto de participantes lo hacen solo a través de sus actos individuales de intercambio. El grupo motor, sin embargo, se queja en ocasiones de la falta de participación y compromiso de otros usuarios, debido a la escasa o nula generación de intercambios por algunas personas de la red, y que atribuyen a las actitudes adquiridas de su experiencia en el mercado, como el miedo a endeudarse (cuentas en negativo) o, simplemente, el uso de la red para su beneficio personal. A este respecto, además, el motivo esgrimido de la falta de tiempo suele ser uno de los más frecuentes.

Más allá del intercambio habitual de bienes y servicios, de manera esporádica se organizan mercadillos donde principalmente se intercambian objetos de segunda mano y de elaboración propia. Estas son ocasiones en las que los miembros pueden reunirse, sociabilizarse y compartir su tiempo libre de una manera más festiva, y donde la organización de tales eventos puede ser remunerada en bellotas.

Una preocupación constante no solo observada en la Bellota, sino en muchos de los grupos de monedas sociales conocidos, nace de la utilización de una cuenta llamada "Administración" para remunerar las tareas que realizan de forma voluntaria algunas de las personas de la red, normalmente el grupo motor. La "deuda" que esta cuenta suele registrar es grande y alerta sobremanera a los participantes, que idean toda una serie de acciones encaminadas a disminuirla. En el caso de la Bellota, y para evitar el agravamiento de esta deuda, se decidió aplicar una tasa del 10\% sobre las transacciones, que iría destinada a la remuneración de estas tareas, que básicamente resumen en: 1) preparación de asambleas y elaboración de actas, 2) gestión de altas y bajas, 3) organización de mercadillos y 4) contabilidad de la Administración.

Este tipo de decisiones genera tensiones en torno a los valores que han de prevalecer en estas experiencias. Por ejemplo, una de las participantes se quejaba recientemente de la tasa antes mencionada: 
Uno de los objetivos de la moneda es facilitar los intercambios y que se pueda triangular y pensar en lo que es el dinero y otro modelo de economía, pero estamos siempre explorando límites, que cuánto dinero puedes tener, que cuánto puedes deber, comisiones, inflaciones... (Conversación en el grupo de WhatsApp del grupo motor de la Bellota, a 13-11-2018).

Dentro de los objetivos que la Bellota se planteaba en sus inicios también estaba favorecer la solidaridad y el apoyo mutuo ${ }^{23}$ entre sus miembros. Así se declaraba a una emisora de radio local por parte de uno de sus miembros: "se busca construir una comunidad basada en el apoyo mutuo, en la confianza y crear relaciones sociales". Esto debería concurrir en una mayor circulación de recursos y en un aumento de la vida comunitaria. Una de las participantes de la red declaraba en una asamblea:

Es prioritario que la gente se vea las caras para que los intercambios fluyan y se cree comunidad. Tanto los mercadillos como las asambleas son para verse, punto de encuentro para conocerse, así es como surgen las necesidades, te conoces y esto facilita el intercambio. Te ves, te relacionas, te pones cara.

Por ello, dentro del grupo motor se perciben los mercadillos como ocasiones clave para la recreación de los vínculos entre participantes, a la vez que estos espacios están frecuentemente presentes en el imaginario colectivo para el fin de crear comunidad. Es significativa la importancia que se da a las relaciones cara a cara y a la creación de espacios de encuentro para favorecer los intercambios, ya que esto se considera el primer paso para cambiar las relaciones económicas.

De algún modo, se observa en la mayoría de monedas sociales la preocupación por promover transacciones económicas basadas en el apoyo mutuo entre personas. La mayoría de participantes reconoce que al entrar a la Bellota no conocía a nadie de la red, por lo que, a priori, las relaciones entre los participantes se parecen más a las relaciones contractuales donde el intercambio se decide libremente (Supiot, citado en Sabaté, 2009). A pesar de ello, se pretende crear vínculos sociales y de reciprocidad en un contexto específico que ayude a cubrir necesidades básicas dentro de una "comunidad" en un contexto de crisis económica y fuerte desempleo. Esta idea aparece como la principal razón de ser para impulsar la iniciativa como se puede ver en el siguiente párrafo de la nota de prensa que se envió a los medios de comunicación para el acto de presentación de esta moneda social en Guadalajara:

Las monedas sociales son un sistema de intercambio de bienes, servicios o saberes, creado y organizado por una comunidad local, basado en la confianza y que se produce mediante la creación de un instrumento de intercambio propio que funciona como unidad de valor. De esta forma, se permite crear una economía

23 En la diversidad de experiencias de moneda social conocidas, se observa la tensión entre los objetivos económicos o sociales, y la definición de modelos de gestión de la moneda. Esta discusión en curso se hace evidente de un modo especial durante los encuentros estatales de monedas sociales. Entre ellas, se reivindican más los objetivos sociales en los modelos denominados de "crédito mutuo", que suelen estar asociadas a la creación de lazos sociales, frente a las que se consideradas más ligadas a objetivos económicos y al respaldo de la economía local, normalmente respaldadas en dinero oficial, y consideradas complementarias. 
complementaria a la convencional, que se basa en la autogestión, el reparto y la cooperación. Las monedas complementarias ayudan, entre otras cosas, a generar nuevos vínculos sociales; ayudan a relocalizar la economía al privilegiar la producción y el consumo local; y son un instrumento útil para luchar contra la pobreza al crear medios adicionales para adquirir bienes y permitir integrar a personas desempleadas en una lógica de intercambio que valoriza sus capacidades productivas (Fragmento de la nota de prensa enviada a los medios de comunicación).

Como vemos, estas redes de intercambio son concebidas como instrumentos para hacer frente a la crisis financiera ${ }^{24}$, porque, por un lado, se vinculan a la recuperación de habilidades personales y profesionales dejadas de lado, dada su desvalorización en los sectores formales de la economía, y por otro, se consideran útiles para la creación de empleo al favorecer la creación y fortalecimiento de contactos (FernándezPacheco, 2017). Se reconoce así que participar en este tipo de sistemas se convierte en un potencial activo que genera capital social para una comunidad, ya que favorece la cohesión social: "este capital social no solo favorece y amplía las posibilidades de empleo a través de la generación de nuevas redes sociales, sino que, además, está contribuyendo al empoderamiento de la comunidad a través de la cohesión social" (Fernández-Pacheco, 2017: 473).

Se supone que, a diferencia del comportamiento económico basado en la lógica del beneficio y la competencia individual, el compromiso de los participantes en el uso de la red intercambio y la comunidad de personas que la integran, favorecerá la transformación de las transferencias en su seno y se reforzarán las relaciones sociales subyacentes, como en la teoría de la reciprocidad (Mauss, 2009).

\section{Discusión: Entre la reciprocidad y el mercado}

Un análisis más profundo de la Bellota nos va a permitir visibilizar algunos aspectos que a priori podrían parecer contradictorios con la aspiración a un modelo de intercambio nuevo donde la lógica del beneficio individual -y el cálculo de equivalencias-no esté presente. Ciertas características de la organización de estas redes parecen mucho más cercanas a la lógica de mercado que a las prácticas desinteresadas de la reciprocidad, como así lo demuestra, por ejemplo, la presencia de un medio de intercambio, la medición en el cálculo de valor en unidades de moneda social y la obligación de reembolso en un tiempo específico, entre otros.

De acuerdo con Sanz (2002), quien realiza un análisis asociado a los BDT, entendemos que ciertas nociones - deuda, inflación o cuentas en negativo- remiten a una dimensión contractual de los intercambios. Por ello, tal vez debamos reconsiderar que la moral de la reciprocidad entra en contradicción con las formas de gestión

\footnotetext{
24 Aunque los objetivos de integración social están presentes en la Bellota, en la práctica se observa que estas redes no se componen de las personas más carentes de recursos económicos. Como señalaba uno de los principales promotores de monedas sociales en España, Julio Gisbert, en el marco del último Encuentro Estatal de Monedas Sociales -en el año 2018-: "esto no es como en Argentina, donde vino el trueque y salvó a millones de personas, no es así (...), desde mi punto de vista, creo que no están para una cuestión económica, sino para una cuestión social de hacer comunidad o hacer vecindad". Este hecho quizás sea debido a la naturaleza mayormente ideológica que se percibe en los objetivos y valores de estos circuitos de intercambio.
} 
basadas en el registro, la medición y, en definitiva, con mecanismos que remiten a la contabilidad bancaria mercantil.

En efecto, el ejemplo aquí considerado no parece que pueda encajar completamente con lo que los antropólogos han definido como reciprocidad entendida en una de sus acepciones más comunes, como "intercambios en los que el cálculo de equivalencias entre bienes y servicios transferidos y los tiempos de cierre de la transacción no quedan definidos de antemano" (Sanz, 2002:154). Asimismo, se supone que ser miembro de estas redes supone un cierto nivel de compromiso personal, pero en la práctica sucede que muchas personas que se adhieren a la red la usan de una manera interesada y eventual, sin participar regularmente en ninguna tarea de gestión ni otro espacio de sociabilidad, tal como mercadillos, asambleas, etc.

Sin embargo, estos sistemas de intercambio no monetario tampoco pueden entenderse, por oposición a los intercambios definidos en el mercado. Entonces, ¿cómo podemos interpretar estos intercambios? Las características complejas de estos sistemas de intercambio requieren un análisis añadido en un marco más amplio de articulación de modalidades de intercambio.

\subsection{Después de la dicotomía clásica. Coexistencia de lógicas económicas}

Polanyi (2016 [1944]) señaló la novedosa ruptura del sistema capitalista respecto a otros sistemas anteriores al desincrustar la economía del resto de instituciones sociales. Desde una perspectiva histórica, el dominio de la lógica del mercado se consolida con el triunfo del sentido formal de la economía sobre el uso sustantivo de la misma en el S.XIX. En esta dirección, hemos visto, la ESS -y sus prácticas correspondientes, las monedas sociales en este caso- pueden comprenderse como intentos de contrarrestar la hegemonía del mercado. Esta organiza el acceso a los bienes y servicios a través del mecanismo del mercado (oferta-demanda-precio), lo que, con base en el poder adquisitivo de las personas, excluye a los más desfavorecidos del acceso a los recursos. En oposición, a través de estas prácticas se pretende ofrecer alternativas para la satisfacción de las necesidades reales de las personas. Aquí está presente el deseo de vincular - o reinsertar- la economía en la sociedad como propone el modelo de la ESS.

En esta dirección, como se ha descrito anteriormente, la ESS basándose en Mauss y Polanyi reproduce un marco dicotómico - y evolutivo- de formas de intercambio reciprocidad/mercado (primitiva/moderna) que no se corresponde con ninguna realidad empírica, más allá del papel dominante de estas modalidades en diferentes momentos. La propuesta fundamental en la mayoría de los trabajos presentes en la ESS es una diferenciación entre dos lógicas económicas contrapuestas que parece apoyarse en una secuencia lineal.

Se podría considerar que el propósito de la Bellota es intervenir en el ámbito de una comunidad potencial de usuarios donde se realizan transacciones a largo plazo orientadas a la reproducción social más allá de la esfera de mercado. Por consiguiente, estas experiencias promueven un ámbito donde las personas puedan satisfacer sus necesidades sin recurrir al dinero, donde la búsqueda de beneficio individual se reduzca, y sea sustituida por una lógica basada en la solidaridad y el reconocimiento mutuo, similar a la de reciprocidad generalizada (Sahlins, 1983). Como resultado, y también debido a la ausencia de una moneda, los bienes y servicios transferidos aquí, 
pueden parecer completamente fuera de la esfera del mercado. Pero un examen más minucioso revela lo inapropiado de esta afirmación.

Se ha señalado desde el inicio la centralidad que ocupa en los objetivos de estas monedas la generación de vínculos sociales. En estas experiencias se demarca una comunidad de participantes ${ }^{25}$ a través de unos límites y normas bien definidas. No obstante, la propia organización y normativa de la red -basada en la cuantificación, la oferta y la demanda de bienes y servicios, etc.- permite las actuaciones basadas en el propio interés. Al ofrecer un bien o servicio algunas personas pueden estar motivadas solamente por la posibilidad de acceder a su vez a otros recursos o ahorrar para conseguir una serie de servicios costosos en el mercado. En la Bellota, la negociación en los precios -y los precios mixtos- están permitidos, especialmente cuando el precio de mercado de los materiales -o los desplazamientos en coche en caso de ser necesarios- se puede incluir en el valor de un servicio prestado. Con ello, dar algo a otro miembro presenta similitudes con un trabajo que se realizaría en el ámbito del mercado para comprar algo con los ingresos resultantes.

Pero incluso si estas circunstancias tienen lugar, no significa que se incumplan las normas establecidas por la comunidad de intercambio, ya que también se observa una cierta permisividad para las transacciones de interés propio dentro de la red, aunque estas provoquen malestar en el resto de participantes involucrados, como cuando alguien trata de convertirse en miembro ofreciendo solo objetos de segunda mano, ya que, como señalase uno de los coordinadores: "la Bellota no se concibe como una forma de sacar rentabilidad a lo que no quieres, no aportar tus habilidades o recursos e inscribirte ofreciendo solo cacharros que tienes ahí" (participante en conversación a través del grupo de Whatsapp de la Bellota, 29-11-2018).

En todo caso, como sucede también cuando las cuentas de algunos/as participantes se encuentran en estado muy negativo -y no se movilizan- se animaría a los miembros a realizar trabajos para la "comunidad", o diversificar su oferta para hacerla más atractiva al resto de participantes. Del mismo modo, si la información referida a una oferta publicada resulta ambigua en relación al precio y servicio ofrecido ${ }^{26}$, se advertiría a la persona correspondiente para dar una mayor claridad al anuncio.

Con todo, se espera que, para los participantes habituales, las transacciones se vuelvan cada vez más desinteresadas a medida que la distancia social se reduce y se establecen relaciones más cercanas -de compañerismo o incluso amistad-. Las motivaciones pueden volverse menos centradas en el interés, y más reorientadas hacia el contacto social con el otro y la formación de una comunidad de ayuda mutua entre individuos dependientes entre sí. Las obligaciones morales compartidas condicionarían el comportamiento de cara al mantenimiento de la comunidad misma por encima del interés propio. En efecto, este es el caso de algunos miembros más comprometidos que, durante largos periodos de tiempo asumen tareas de coordinación y gestión de forma voluntaria, ya que se sienten responsables de que el proyecto funcione, y rechazan ser remuneradas en bellotas por la realización de las mismas, como así lo manifestaba una de las participantes ante la aprobación de la tasa que

25 Cabe señalar que, aunque el requisito para la participación en la Bellota pase por convertirse en miembro de la comunidad, los participantes no siempre se conocen entre sí. También sucede, que estos pueden abandonar la red en cualquier momento si lo desean, ya que su compromiso con la misma, aunque es una condición previa para la participación, en la práctica se observa que no es una cuestión principal.

26 Ocurre en ocasiones, que algunas ofertas pueden expresar el valor íntegramente en bellotas, y a la hora de concretarse la transacción, el valor incluye dinero convencional. 
hemos mencionado anteriormente para remunerar las actividades: "es como si yo cobrase por participar en el AMPA del colegio, yo hago esto porque quiero, de manera voluntaria, lo hago por el bien común" (participante en conversación, 5/12/18).

A este respecto, nos parece muy ilustrativo un ejemplo que nos relataba la coordinadora de otra red de intercambio, sobre una socia que llevaba muchos años enferma. Estaba sola porque su hijo vivía fuera, y un grupo muy reducido de la red estuvo haciendo turnos en el hospital para ayudarla. Finalmente murió, y pasó lo siguiente:

Su hijo no trabajaba (...), no tenía dinero, incineraron a su madre y nos enteramos por el grupo más allegado que él solo tenía $1000 €$ y necesitaba $600 €$ más, entonces una turutaira le dejó dinero, le dijo, ya me lo devolverás. Entonces dijimos, si nosotros somos un grupo de ayuda mutua y nosotros somos una comunidad, si R. ha estado siempre ahí, en las fiestas y las reuniones, nosotros debemos ayudar a R., aunque ya no esté. Hicimos un llamamiento a todos los turutairas, aunque no la conocían pusieron dinero, en tres semanas conseguimos $1600 €$ que le dimos a la señora que había puesto el dinero (Celia, entrevista 26/10/18).

Por consiguiente, es cierto que los participantes más comprometidos ocasionalmente se ven envueltos en prácticas de ayuda mutua desinteresada entre sí. El hecho de que las personas permanezcan en la red después de cada transacción concreta, hace que los vínculos tiendan a reforzarse después de cada encuentro y adquieran una orientación a largo plazo vinculada con la reproducción social (Bloch y Parry, 1989). Con ello, las decisiones supuestamente contractuales y libres tomadas por las personas en las transacciones iniciales pueden transformarse en una implicación con otros participantes a medida que se reduce la distancia social entre ellos. A pesar de ello, esta construcción de vínculos sociales -más allá de casos eventuales como el descrito más arriba- suele limitarse normalmente a las relaciones de uno a uno, por lo que no podría decirse que hay una construcción de comunidad en el anteriormente mencionado, sino simplemente de vínculos personales. Entretanto, la unión de una persona a todo el grupo de participantes puede mantenerse impersonal a lo largo del tiempo, aun si se organizan mercadillos, fiestas y asambleas, donde cabría esperar que se intensificase el sentido de comunidad entre los miembros.

Aunque es obvio que no se puede decir que las transacciones presentes en la Bellota ocurran en el ámbito del mercado, tampoco parece que puedan ajustarse completamente a la definición de reciprocidad de Mauss. Por ello, lo que hemos propuesto aquí, es que lejos de entender estas redes de intercambio y la esfera del mercado como dos compartimentos estancos, vale la pena examinar su articulación. Del mismo modo, del análisis de estas experiencias se puede desprender cómo en los significados y valores que se otorgan a los bienes y servicios a transferir, confluyen cuestiones que tienen que ver con aspectos tanto económicos, como sociales, culturales e ideológicos.

\section{Conclusiones}

E1 material etnográfico presentado aquí demuestra que la noción antropológica de reciprocidad plantea inconvenientes a la hora de ajustarse totalmente al tipo de rela- 
ciones económicas no comerciales que pretenden promover las monedas sociales. El objetivo principal de estos proyectos, como experiencias específicas de la ESS, es reintegrar la circulación económica a las necesidades sociales ofreciendo alternativas al mercado, por lo que se ha examinado aquí el papel de la noción de reciprocidad en la definición de sus objetivos y en las transacciones. Pero remitirnos en el análisis a una interpretación de la noción de reciprocidad a la manera sugerida por los autores clásicos, no podría haber tenido mucho recorrido, por lo que hemos señalado la necesidad de un examen más detallado del tipo de relaciones subyacentes en estas experiencias.

Por un lado, la experiencia apunta a una des-mercantilización de las prácticas económicas al haber sido ubicadas por sus promotores, a propósito, fuera de la esfera del mercado. En este sentido, dan valor al trabajo, más allá de su remisión al mercado laboral -reconociendo las habilidades y capacidades de trabajo de las personas a pesar del desempleo en un contexto de crisis- y consideran las necesidades de las personas como algo prioritario que no es tratado al modo del mercado, como simples demandas.

Sin embargo, incluso si verdaderamente se sitúan en el ámbito de la comunidad, no pueden ser consideradas como manifestaciones indiscutibles de la "reciprocidad" tal y como es considerada en el universo maussiano, puesto que las obligaciones de dar, regalar y devolver solo surgen eventualmente, como hemos visto. Además, puede influir el hecho de que a priori estas experiencias están enmarcadas en un contexto de adscripción voluntaria y no de obligación moral.

Quizás también puede influir que la participación no depende en gran medida de las conexiones sociales anteriores, dado que la mayoría de personas no se conoce previamente al entrar en la red. No obstante, después de un tiempo de participación, una cierta restricción de la autonomía personal puede resultar de transacciones repetidas, incluso si cada decisión sobre la participación sigue siendo libre, y la red puede abandonarse en cualquier momento. En este sentido, un marco moral similar a la comunidad no puede alcanzarse por completo, pero algunas obligaciones, similares a las que existen en contextos menos electivos como el vecindario, pueden surgir de las demandas y expectativas de los amigos que también participan. Por tanto, se observa que a veces hay lugar para prácticas desinteresadas entre los participantes más comprometidos.

Pero, para determinar el alcance particular de estas iniciativas $-\mathrm{y}$ el valor o no del concepto de reciprocidad para el análisis de estas prácticas-, se necesitaría una mayor profundización tanto teórica como empírica. En este último caso, si se valida que para los participantes prevalece la búsqueda del contacto social sobre el cálculo y el propio interés, entonces se podrá decir que han logrado su objetivo de compensar hasta cierto punto la omnipresencia de la norma del mercado.

\section{Bibliografía}

Alquézar, Raquel (2015) La economía social y solidaria y las finanzas éticas: entre el valor social y el valor de mercado. Tesis doctoral. Facultat de Geografía i História. Universitat de Barcelona.

Bohannan, Paul (1981) "El impacto de la moneda en una economía africana de subsistencia". En Llobera (comp.) Antropología Económica. Barcelona. Anagrama, 189-200 
Blanc, Jerome (2011) “Classifying 'CCs': Community, Complementary and Local Currencies”. International Journal of Community Currency Research, $\mathrm{n}^{\circ} 15$ (D), pp. 4-10

Bloch, Maurice y Parry, Jonathan (1989) Money and the morality of exchange. Cambridge: Cambridge University Press.

Corrons, August (2015) Monedas complementarias en pro de la sostenibilidad y el desarrollo: enfoque panárquico. Trabajo de investigación del máster interuniversitario en cooperación al desarrollo. Universitat Jaume I.

Fernández-Pacheco, José Luis (2017) Estrategias de Desarrollo Local frente a la crisis en entornos rurales vulnerables: una comparativa de casos entre sudáfrica y España. Tesis doctoral. Facultad de Ciencias Políticas y Sociología. Universidad Complutense de Madrid.

Gisbert, Julio (2010) Vivir sin empleo. Barcelona: Los libros del lince.

Godelier, Maurice (2004) "Acerca de las cosas que se dan, de las cosas que se venden y de las que no hay que vender ni dar, sino que hay que guardar: una reevaluación crítica del ensayo sobre el don de Marcel Mauss", en Paz Moreno (ed.), Entre las gracias y el molino satánico: lecturas de Antropología Económica. Madrid: UNED, 195-210

Hart, Keith (2001) The memory bank: Money in an unequal world. Londres: Profile Books.

Harvey, David (2004) El nuevo imperialismo, Madrid: Akal

Lanchester, John (2016) "When bitcoin grows up". London Review of Books, 38(8), 3-12

Laville, Jean-Louis (2004) "El marco conceptual de la economía solidaria”, en Laville JeanLouis (ed.), Economía social y solidaria. Una visión europea, Buenos Aires: Altamira, pp. 207-235.

Lietaer, Bernand (2005) El futuro del dinero. Buenos Aires: Errepar/Longseller editores

Lietaer, Bernard; Kennedy, Margrit; Rogers, John (2015) El dinero de la gente. Monedas locales y soberanía económica. Barcelona: Icaria

Malinowski, Bronislaw (1986 [1922]) Los argonautas del pacifico occidental. Barcelona: Península

Mauss, Marcel (2009) Ensayo sobre el don. Forma y función del intercambio en las sociedades arcaicas. Buenos Aires: Katz Editores.

Moreno, Paz (2011) El bosque de las Gracias y sus pasatiempos: Raíces de la antropología económica. Madrid: Editorial Trotta.

Narotzky, Susana (2010) "Reciprocidad y capital social: modelos teóricos, políticas de desarrollo, economías alternativas. Una perspectiva antropológica”. En Bretón, V. Saturno devorando a sus hijos: miradas críticas sobre el desarrollo y sus promesas. Barcelona: Icaria Desarrollo Rural.

Narotzky, Susana (2016) “El proyecto en el modelo: Reciprocidad, capital social y la política del realismo etnográfico. (Con)textos.. Revista d'antropologia i investigació social, $\mathrm{n}^{\mathrm{0}}$ 6, 84-99

Oliver, Esther (2013) Monedas sociales en España: estado actual y aportaciones a la sostenibilidad de los sistemas de intercambio comunitario en España. UNED-UJI

Orzi, Ricardo (2009) El potencial emancipador de la Moneda Social en la construcción de subsistemas de Economía Social y Solidaria (ESS): una consideración sobre el concepto de Moneda Social a partir de una revisión crítica sobre los enfoques tradicionales de la moneda: la moneda como lazo social. Disponible en: http:/www.econ.uba.ar/seminario/ Ponencias/Eje\%206/El\%20potencial\%20emancipador\%20de\%201a\%20Moneda\%20 Social-Orzi.pdf.

Primavera, Heloisa (1999) La moneda social de la red global de trueque en Argentina. ¿Barajar y dar de nuevo en el juego social? Disponible en www.socioeco.org/bdf_fichedocument-1251_es.html 
Polanyi, Karl (2016 [1944]) La Gran Transformación. Crítica del liberalismo económico. Barcelona: Virus

Putman, Robert (1993) Making democracy work. Civic traditions in modern Italy. Princeton: Princeton University Press.

Sabaté, Irene (2009) "Exchange networks and free shops in Berlin: gifts and commodities in 'alternative' consumption experiences". Etnográfica. 13 (1) pp 49-75.

Sahlins, Marshal (1983), Economía de la Edad de Piedra. Madrid: Akal

Santana, Ma Eugenia (2009) Reinventando el dinero. Experiencias con monedas comunitarias. http://docplayer.es/2949744-Reinventando-el-dineroexperiencias-con-monedascomunitarias.html

Sanz, Gonzalo (2002) "Las asociaciones de banco de tiempo: entre la reciprocidad y el mercado". Éndoxa: Series Filosóficas, n 15, pp. 153-163: UNED Madrid

Seyfang, Gill y Longhurst, Noel (2013) "Growing green money? Mapping community currencies for sustainable development". Ecological economics. 86, pp 65-77

Yasuyuki, Miguel (2017) Monedas sociales y complementarias (MSCs): sus valores socioeconómicos para distintos stakeholders. (Tesis doctoral), Universitat de Valéncia

Zelizer, Viviana (2015). Vidas económicas. Cómo la cultura da forma a la economía. Madrid: Centro de Investigaciones Sociológicas. 